5. Клочков Ю. В., Николаев А. П., Киселева Т. А. Анализ НДС произвольной непологой оболочки в форме компенсатора с использованием векторной интерполяции полей перемещений // Изв. Волгоград. техн. ун- та : межвуз. сб. науч. ст. № 10(97)/ ВолгГТУ. Волгоград : ИУНЛ ВолгГТУ, 2012 (Сер. Актуальные проблемы управления, вычислительной техники и информатики в технических системах. Вып. 14). С. 28-32.

\title{
Stress-strain State of an Elliptical Cylinder with an Ellipsoidal Bottoms of Dissimilar Materials Based FEM
}

\author{
J. V. Klochkov, A. P. Nikolaev, T. A. Kiseleva
}

Volgograd State Agricultural University, Russia, 400002,University av., 26, Klotchkov@ @k.ru, anpetr40@yandex.ru, kiseleva_ta@ro.ru

The algorithm of calculating the construction in the form of an elliptical cylinder with ellipsoidal bottom of different materials based on the finite element method with the use of scalar and vector fields interpolating movements is described. As part of the sampling using rectangular curved finite elements with eighteen degrees of freedom in the node. Calculations of a circular cylinder with an articulated ellipsoid of rotation the verification of the algorithm and shows its effectiveness.

Key words: articulated shell, scalar interpolation, vector interpolation, rectangular finite element, ellipsoid, cylinder.

\section{References}

1. Klochkov J. V., Nikolaev A. P., Kiseleva T. A. Comparison of options interpolations movement as an example of an arbitrary shell in the shape of an ellipsoid. Vestnik Volgogradskogo Gos. Arch.- Stroit. Univ. Ser. Str-vo i Arhit. [Bulletin of the Volgograd State Architectural and Building Univ. Ser. The Construction and Arch.], 2011, no. 23(42), pp. 54-59 (in Russian).

2. Nikolaev A. P., Klochkov J. V., Kiselev A. P., Gureeva N. A. Vektornaja interpoljacija polej peremeshhenij o konechno-jelementnyh raschetah [Vector interpolation displacement fields in finite-element calculations]. Volgograd, 2012, 264 p. (in Russian).

3. Sedov L. I. Mekhanika sploshnoi sredy [Continuum Mechanics]. Moscow, Nauka, 1976, vol. 1, 536 p. (in Russian).
4. Postnov V. A., Harhurim I. J. Metod konechnykh elementov v raschetakh sudovykh konstruktsii [The Finite Element Method in the Calculation of Ship Structures]. Leningrad, Sudostroenie, 1974. 344 p. (in Russian).

5. Klochkov J. V., Nikolaev A. P., Kiseleva T. A. Analysis VAT Arbitrary Nonshallow Shell in the Form of the Compensator Using Vector Interpolation of Displacement Fields. Izvestiya Volgogradskogo Texniheskogo Universiteta [Proceedings of the Volgograd Technical University]: Interuniversity. Sat Scientific. Art. no. 10 (97) / VolgGTU. Volgograd IUNL VolgGTU, 2012 (Ser. Actual problems of management, computer science and informatics in technical systems. iss. 14), pp. 28-32 (in Russian).

\section{ЗАДАЧИ ГИДРОУПРУГОСТИ ДЛЯ ТРУБЫ КОЛЬЦЕВОГО СЕЧЕНИЯ С УПРУГОЙ, ГЕОМЕТРИЧЕСКИ НЕРЕГУЛЯРНОЙ ВНЕШНЕЙ ОБОЛОЧКОЙ ПРИ ВОЗДЕЙСТВИИ ДАВЛЕНИЯ}

\author{
Д. В. Кондратов ${ }^{1}$, Ю. Н. Кондратова ${ }^{2}$, В. С. Попов ${ }^{3}$, И. В. Плаксина ${ }^{4}$
}

\footnotetext{
${ }^{1}$ Доктор фризико-математических наук, заведующий кафредрой прикладной инфрорматики и инфрормационных технологий в управлении, Поволжский институт управления им. П. А. Столыпина, Саратов, KondratovDV @yandex.ru

${ }^{2}$ Кандидат физико-математических наук, доцент кафредры математической кибернетики и компьютерных наук, Саратовский государственный университет им. Н. Г. Чернышевского, KondratovaUN@ info.sgu.ru

${ }^{3}$ Доктор технических наук, заведующий касредрой теплогазоснабжения, вентиляции, водообеспечения и прикладной гидрогазодинамики, Саратовский государственный технический университет им. Гагарина Ю. А., vic_p@bk.ru ${ }^{4}$ Преподаватель кафредры прикладной инсрорматики и инсрормационных технологий в управлении, Поволжский институт управления им. П. А. Столыпина, Саратов, chefirina@yandex.ru
} 
Рассмотрена механическая модель, представленная в виде трубы кольцевого сечения, образованная двумя поверхностями соосных цилиндрических оболочек, взаимодействующими с вязкой несжимаемой жидкостью. Построена математическая модель этой системы, состоящая из дифрференциальных уравнений в частных производных, описывающих динамику вязкой несжимаемой жидкости и упругой ребристой оболочки совместно с граничными условиями.

Ключевые слова: гидроупругость, вязкая жидкость, труба кольцевого сечения, геометрически нерегулярная оболочка.

В настоящее время в различных областях техники используются такие современные конструкции, которые представляют собой сложные системы, описывающие взаимодействия твердых, упругих и жидких тел. Такие конструкции могут быть описаны моделью, состоящей из двух цилиндрических оболочек, вложенных друг в друга, между которыми расположена жидкость. Примерами использования таких моделей с двумя цилиндрическими оболочками могут служить двигатели внутреннего сгорания, поплавковые приборы навигации, жидкостные ракетные двигатели [1-5]. В таких моделях жидкость между оболочками может служить не только для демпфирования собственных колебаний оболочек, но и для охлаждения этих оболочек. Кроме того, внешняя оболочка может быть геометрически нерегулярной, а внутренняя - абсолютно жесткой.

Рассмотрим механическую модель, представляющую собой круглую трубу кольцевого сечения, образованную двумя поверхностями соосных цилиндрических оболочек, взаимодействующими с вязкой несжимаемой жидкостью. Такая система представлена на рис. 1.

Между цилиндрическими оболочками рассматривается ламинарное течение вязкой несжимаемой жидкости, причем внешняя оболочка является упругой геометрически нерегулярной, а внутренняя оболочка является абсолютно жестким цилиндром. Ширина $h_{0}$ цилиндрической щели кольцевого сечения значительно меньше внешнего радиуса $R_{2}$ внутренней оболочки и внутреннего радиуса $R_{1}$ и ра-

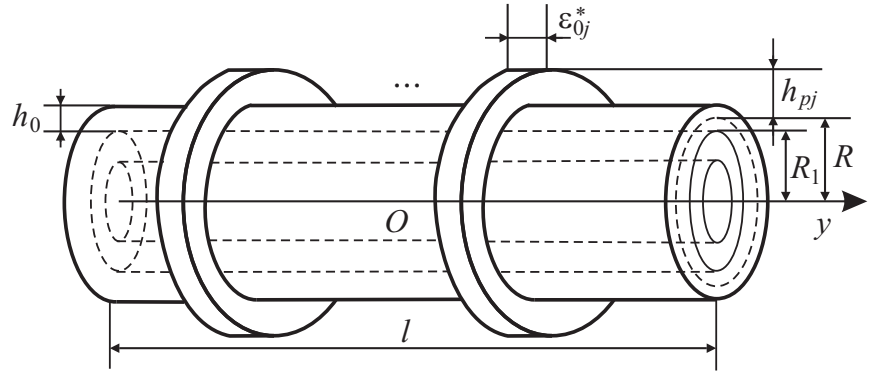

Рис. 1. Механическая модель диуса срединной поверхности $R$ внешней оболочки $h_{0}=2\left(R-R_{1}\right)<<R$. Радиус срединной поверхности $R$ значительно больше толщины внешней $h_{0}=2\left(R-R_{1}\right)$ оболочки. Радиус срединной поверхности оболочки равен $R$, а ее толщина на участках, где отсутствуют ребра жесткости, равна $h_{0}$. Длины цилиндрических оболочек $l$ - одинаковые, а упругие перемещения внешней оболочки значительно меньше ширины $\delta$ цилиндрической щели. Течение жидкости происходит под действием переменного по времени перепада давления. Перемещение внутренней оболочки относительно внешней на торцах отсутствует. Механическая система считается термостабилизированной.

Рассмотрим систему координат $O_{1} x_{1} y_{1} z_{1}$ и свяжем ее с основанием, к которому крепится механическая система. Центр системы $O_{1}$ расположен в геометрическом центре соосных оболочек в невозмущенном состоянии. Будем полагать, что перемещения вдоль оси $O_{1} y_{1}$ отсутствуют. Рассмотрим дополнительно необходимую далее цилиндрическую систему координат $r, \theta, y\left(\bar{n}_{r}, \bar{n}_{\theta}, \bar{j}-\right.$ орты цилиндрической системы). Полюс цилиндрической системы координат совпадает с началом координат $O_{1} x_{1} y_{1} z_{1}$ и направления осей $O y, O_{1} y_{1}$ цилиндрической и декартовой систем координат совпадают (рис. 2).

Внешняя поверхность внешней оболочки трубы является геометрически нерегулярной и имеет $n$ ребер жесткости ступенчато изменяющейся высоты. Ребра представляют собой внешние шпангоуты. Крепление геометрически нерегулярной оболочки на торцах имеет свободное опирание. Ребра жесткости характеризуются своей высотой $h_{p j}$, длиной $\varepsilon_{0 j}^{*}$ и продольной координатой начала ребра $y_{j}$. При этом высота ребра при движении вдоль оболочки изменяется скачкообразно. Нормальная к координатной поверхности координата $z$, внутренней поверхности оболочки постоянна $z_{1}=-h_{0} / 2$.

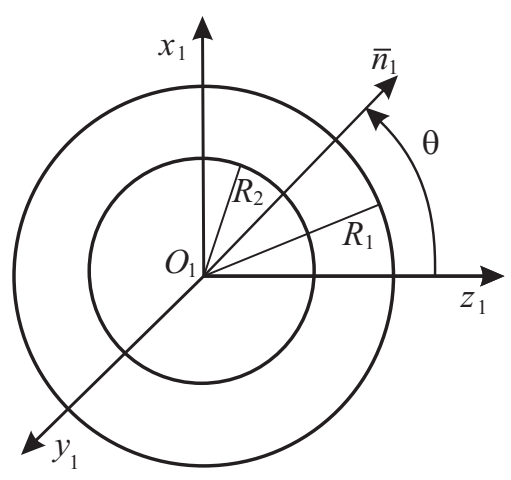

Рис. 2. Системы координат 
Часть внешней поверхности оболочки постоянна $z_{2}=h_{0} / 2$, а расположенные вдоль оси $O y$ в точках $y_{j}(j=1,2, \ldots, n)$ ребра ограничены по высоте поверхностями

$$
z_{2}=h_{p j}-h_{0}=\left(1-\frac{h_{0}}{h_{p j}}\right) h_{p j} .
$$

Таким образом, получается, что внешняя оболочка имеет разрывы в точках оси Оу и в связи с этим возникает трудность в ее описании. Можно воспользоваться единичной функцией Хевисайда $\Gamma(y)$, которая определяется как

$$
\Gamma(y)= \begin{cases}0, & \text { если } y<0, \\ 1, & \text { если } y \geq 0 .\end{cases}
$$

Тогда ступенчатый характер изменения высоты ребра можно описать с помощью разностей функций Хевисайда по продольной координате. Тогда внешнюю поверхность оболочки можно описать с помощью общего уравнения:

$$
z_{2}=\frac{h_{0}}{2}+\sum_{j=1}^{n}\left(1-\frac{h_{0}}{h_{p j}}\right) h_{p j} \Delta \Gamma_{y j},
$$

где $\Delta \Gamma_{y j}=\Gamma\left(y-y_{j}\right)-\Gamma\left(y-y_{j}-\varepsilon_{0 j}^{*}\right), \Gamma(y)-$ единичная функция Хевисайда по продольной координате; $y_{j}$ - точка появления ребра по продольной координате.

Математическая модель рассматриваемой механической системы состоит из дифференциальных уравнений в частных производных описывающих динамику вязкой несжимаемой жидкости и упругой ребристой оболочки совместно с граничными условиями. Наличие жидкости в системе приводит к быстрому затуханию собственных колебаний, и переходный процесс можно не рассматривать, поэтому рассматриваются только вынуждение колебания.

Течение вязкой несжимаемой жидкости между цилиндрическими оболочками осесимметричное и описывается уравнениями Навье-Стокса. В цилиндрической системе координат эти уравнения имеют следующий вид:

$$
\begin{gathered}
\frac{\partial V_{k}}{\partial t}+V_{r} \frac{\partial V_{k}}{\partial r}+V_{y} \frac{\partial V_{k}}{\partial y}=-\frac{1}{\rho} \frac{\partial p}{\partial k}+\nu\left(\frac{\partial^{2} V_{k}}{\partial r^{2}}+\frac{1}{r} \frac{\partial V_{k}}{\partial r}+\frac{\partial^{2} V_{k}}{\partial y^{2}}-\chi \frac{V_{r}}{r^{2}}\right), \\
\frac{\partial V_{r}}{\partial r}+\frac{V_{r}}{r}+\frac{\partial V_{y}}{\partial y}=0 .
\end{gathered}
$$

Здесь $k=r$ или $y$; $\chi=1$ при $k=r, \chi=0$ при $k=y ; V_{y}, V_{r}$ - компоненты вектора скорости жидкости в цилиндрической системе координат $\left(\bar{n}_{r}, \bar{j}\right)$, начало $O$ которой находится в центре внутренней оболочки; $p$ - давление жидкости; $\rho$ - плотность жидкости; $\nu$ - кинематический коэффициент вязкости; $y$ - координата вдоль оси симметрии $O y ; r-$ расстояние от оси $O y$; $t$ - время.

Граничные условия для системы (1) представляют собой условия прилипания вязкой жидкости к поверхностям оболочек и условия для давления на концах механической системы:

$$
V_{r}=\frac{\partial u_{3}}{\partial t}, \quad V_{y}=-\frac{\partial u_{1}}{\partial t} \quad \text { при } \quad r=R_{2}+\delta+u_{3}^{\prime}
$$

$V_{r}=0, V_{y}=0$ при $r=R_{2} ; p=p^{+}$при $y=l / 2, p=p^{-}$при $y=-l / 2$, где $u_{3}-$ прогиб внешней оболочки, положительный в сторону, противоположную центру кривизны; $u_{1}$ - продольное перемещение оболочек положительное, в сторону противоположную оси $O y$.

Для вывода уравнений динамики ребристой оболочки применим вариационный интегральный принцип Гамильтона. Принцип Гамильтона в цилиндрической системе координат можно записать в виде

$$
\begin{gathered}
\delta \int_{t_{0}}^{t_{1}}[L+(\bar{q}, \bar{u})] d t=0 \\
L=\frac{1}{2} \int_{z_{1}}^{z_{2}} \iint_{\Omega}\left[\rho_{0}\left(\frac{\partial \bar{u}^{z}}{\partial t}, \frac{\partial \bar{u}^{z}}{\partial t}\right)-\left(\sigma_{s} \varepsilon_{s}^{z}+\sigma_{\theta} \varepsilon_{\theta}^{z}+\tau_{s \theta} \gamma_{y \theta}^{z}\right)\right] d z d \Omega,
\end{gathered}
$$


где $L-$ функция Лагранжа; $\bar{q}-$ вектор поверхностных усилий; $\bar{u}=u_{1} \bar{n}_{y}+u_{2} \bar{n}_{\theta}+u_{3} \bar{n}-$ вектор упругих перемещений координатной поверхности оболочки $\left(\bar{n}_{y}, \bar{n}_{\theta}, \bar{n}-\right.$ продольное и окружное направления в координатной поверхности оболочки и нормаль к ней); $\rho_{0}$ - плотность материала оболочки; $\bar{u}^{z}$ - вектор упругих перемещений точек оболочки, отстоящих от координатной поверхности на расстоянии $z ; \sigma_{y}, \sigma_{\theta}, \tau_{y \theta}-$ компоненты тензора напряжений; $\varepsilon_{y}^{z}, \varepsilon_{\theta}^{z}, \gamma_{y \theta}^{z}-$ компоненты тензора упругой деформации; $d \Omega=R d \theta d s$.

Согласно гипотезам Кирхгофа-Лява имеем:

$$
\begin{gathered}
\bar{u}=u_{1}^{z} \bar{n}_{y}+u_{2}^{z} \bar{n}_{\theta}+u_{3}^{z} \bar{n}, \\
u_{1}^{z}=u_{1}-z \frac{\partial u_{3}}{\partial y}, \quad u_{2}^{z}=u_{2}-z\left(\frac{1}{R} \frac{\partial u_{3}}{\partial \theta}-\frac{u_{2}}{R}\right), \quad u_{3}^{z}=u_{3}, \\
\sigma_{y}=\frac{E}{1-\mu_{0}^{2}}\left(\varepsilon_{y}^{z}+\mu_{0} \varepsilon_{\theta}^{z}\right), \quad \sigma_{\theta}=\frac{E}{1-\mu_{0}^{2}}\left(\varepsilon_{\theta}^{z}+\mu_{0} \varepsilon_{y}^{z}\right), \quad \tau_{y \theta}=\frac{E}{2\left(1+\mu_{0}\right)} \gamma_{y \theta}^{z}, \quad \varepsilon_{y}^{z}=\frac{\partial u_{1}}{\partial y}-z \frac{\partial^{2} u_{3}}{\partial y^{2}}, \\
\varepsilon_{\theta}^{z}=\frac{1}{R} \frac{\partial u_{2}}{\partial \theta}+\frac{u_{3}}{R}-z \frac{1}{R^{2}}\left(\frac{\partial^{2} u_{3}}{\partial \theta^{2}}-\frac{\partial u_{2}}{\partial \theta}\right), \quad \gamma_{y}^{z}=\frac{1}{R} \frac{\partial u_{1}}{\partial \theta}+\frac{\partial u_{2}}{\partial y}-z \frac{2}{R}\left(\frac{\partial^{2} u_{3}}{\partial y \partial \theta}-\frac{\partial u_{2}}{\partial y}\right),
\end{gathered}
$$

где $E$ - модуль Юнга материала оболочки; $\mu_{0}-$ коэффициент Пуассона.

Уравнение (2) для оболочки $(-l / 2 \leq y \leq l / 2,0 \leq \theta \leq 2 \pi)$ после преобразования функции Лагранжа (3) согласно (4) и интегрирования по $z$ запишется в виде

$$
\begin{aligned}
& \int_{t_{0}}^{t_{1}} \iint_{\Omega}\left\{\left[R \frac{\partial N_{1}^{p}}{\partial y}+\frac{\partial S^{p}}{\partial \theta}+R q_{y}-R h_{0} \rho_{0} \frac{\partial^{2} u_{1}}{\partial t^{2}}\left(1+\sum_{j=1}^{n} k_{1 j} \Delta \Gamma_{y j}\right)\right] \delta u_{1}+\right. \\
& +\left[R \frac{\partial S^{p}}{\partial y}+\frac{\partial N_{2}^{p}}{\partial \theta}+\frac{1}{R} \frac{\partial M_{2}^{p}}{\partial \theta}+2 \frac{\partial H^{p}}{\partial y}+R q_{\theta}-R h_{0} \rho_{0} \frac{\partial^{2} u_{2}}{\partial t^{2}}\left(1+\sum_{j=1}^{n} k_{1 j} \Delta \Gamma_{y j}\right)\right] \delta u_{2}+ \\
& \left.+\left[R \frac{\partial^{2} M_{1}^{p}}{\partial y^{2}}+\frac{1}{R} \frac{\partial^{2} M_{2}^{p}}{\partial \theta^{2}}+2 \frac{\partial H^{p}}{\partial y \partial \theta}-N_{2}^{p}+R q_{n}-R h_{0} \rho_{0} \frac{\partial^{2} u_{3}}{\partial t^{2}}\left(1+\sum_{j=1}^{n} k_{1 j} \Delta \Gamma_{y j}\right)\right] \delta u_{3}\right\} d y d \theta d t- \\
& -\int_{t_{0}}^{t_{1}} \int_{0}^{2 \pi}\left[R N_{1}^{p} \delta u_{1}+\left(R S^{p}+H^{p}\right) \delta u_{2}+\left(R \frac{\partial M_{1}^{p}}{\partial y}+2 \frac{\partial H^{p}}{\partial \theta}\right) \delta u_{3}-R M_{1}^{p} \delta\left(\frac{\partial u_{3}}{\partial y}\right)\right]_{-l / 2}^{l / 2} d \theta d t- \\
& -\int_{t_{0}}^{t_{1}} \int_{-l / 2}^{l / 2}\left[S^{p} \delta u_{1}+\left(N_{2}^{p}+\frac{1}{R} M_{2}^{p}\right) \delta u_{2}+\left(\frac{1}{R} \frac{\partial M_{2}^{p}}{\partial \theta}+2 \frac{\partial H^{p}}{\partial y}\right) \delta u_{3}-\frac{1}{R} M_{2}^{p} \delta\left(\frac{\partial u_{3}}{\partial \theta}\right)\right]_{0}^{2 \pi} d y d t+ \\
& +\left.\left.\int_{t_{0}}^{t_{1}} 2 H^{p} \delta u_{3}\right|_{0} ^{2 \pi}\right|_{-l / 2} ^{l / 2} d t+R h \iint_{\Omega}\left[\left(\rho_{0} \frac{\partial u_{1}}{\partial t} \delta u_{1}+\rho_{0} \frac{\partial u_{2}}{\partial t} \delta u_{2}+\rho_{0} \frac{\partial u_{3}}{\partial t} \delta u_{3}\right) \times\right. \\
& \left.\times\left(1+\sum_{j=1}^{n} k_{1 j} \Delta \Gamma_{y j}\right)\right]_{t_{0}}^{t_{1}} d y d \theta=0
\end{aligned}
$$

где

$$
\begin{gathered}
S^{p}=\frac{E h_{0}}{2\left(1+\mu_{0}\right)}\left\{\gamma_{y \theta}\left(1+\sum_{j=1}^{n} k_{1 j} \Delta \Gamma_{y j}\right)+2 \kappa_{y \theta} h_{0} \sum_{j=1}^{n} k_{2 j} \Delta \Gamma_{y j}\right\} ; \\
H^{p}=\frac{E h_{0}^{3}}{12\left(1+\mu_{0}\right)} \kappa_{y \theta}\left(1+\sum_{j=1}^{n} k_{3 j} \Delta \Gamma_{y j}\right)+\frac{E h_{0}}{2\left(1+\mu_{0}\right)} \gamma_{y \theta} h_{0} \sum_{j=1}^{n} k_{2 j} \Delta \Gamma_{y j} ; \\
N_{1}^{p}=\frac{E h_{0}}{1-\mu_{0}^{2}}\left\{\left(\varepsilon_{y}+\mu_{0} \varepsilon_{\theta}\right)\left(1+\sum_{j=1}^{n} k_{1 j} \Delta \Gamma_{y j}\right)+\left(\kappa_{y}+\mu_{0} \kappa_{\theta}\right) h_{0} \sum_{j=1}^{n} k_{2 j} \Delta \Gamma_{y j}\right\} ;
\end{gathered}
$$




$$
\begin{gathered}
N_{2}^{p}=\frac{E h_{0}}{1-\mu_{0}^{2}}\left\{\left(\varepsilon_{\theta}+\mu_{0} \varepsilon_{y}\right)\left(1+\sum_{j=1}^{n} k_{1 j} \Delta \Gamma_{y j}\right)+\left(\kappa_{\theta}+\mu_{0} \kappa_{y}\right) h_{0} \sum_{j=1}^{n} k_{2 j} \Delta \Gamma_{y j}\right\}, \\
M_{1}^{p}=\frac{E h_{0}^{3}}{12\left(1-\mu_{0}^{2}\right)}\left(\kappa_{y}+\mu_{0} \kappa_{\theta}\right)\left(1+\sum_{j=1}^{n} k_{3 j} \Delta \Gamma_{y j}\right)+\frac{E h_{0}}{1-\mu_{0}^{2}}\left(\varepsilon_{y}+\mu_{0} \varepsilon_{\theta}\right) h_{0} \sum_{j=1}^{n} k_{2 j} \Delta \Gamma_{y j}, \\
M_{2}^{p}=\frac{E h_{0}^{3}}{12\left(1-\mu_{0}^{2}\right)}\left(\kappa_{\theta}+\mu_{0} \kappa_{y}\right)\left(1+\sum_{j=1}^{n} k_{3 j} \Delta \Gamma_{y j}\right)+\frac{E h_{0}}{1-\mu_{0}^{2}}\left(\varepsilon_{\theta}+\mu_{0} \varepsilon_{y}\right) h_{0} \sum_{i=1}^{n} k_{2 j} \Delta \Gamma_{y i}, \\
k_{1 j}=\left(1-\frac{h_{0}}{h_{p j}}\right) \frac{h_{p j}}{h_{0}}, k_{2 j}=\left(1-\frac{h_{0}}{h_{p j}}\right) \frac{h_{p j}^{2}}{2 h_{0}^{2}}, \quad k_{3 j}=\left(1-\frac{h_{0}}{h_{p j}}\right)\left(4-2 \frac{h_{0}}{h_{p j}}+\frac{h_{0}^{2}}{h_{p j}^{2}}\right) \frac{h_{p j}^{3}}{h_{0}^{3}},
\end{gathered}
$$

где компоненты деформации координатной поверхности оболочки $\varepsilon_{y}, \varepsilon_{\theta}, \gamma_{y \theta}, \kappa_{y}, \kappa_{\theta}, \kappa_{y \theta}$ связаны с компонентами вектора перемещений координатной поверхности оболочки следующими соотношениями:

$$
\begin{array}{ccc}
\varepsilon_{y}=\frac{\partial u_{1}}{\partial y}, \quad \varepsilon_{\theta}=\frac{1}{R}\left(\frac{\partial u_{2}}{\partial \theta}+u_{2}\right), & \gamma_{y \theta}=\frac{\partial u_{2}}{\partial y}+\frac{1}{R} \frac{\partial u_{1}}{\partial \theta}, \quad \kappa_{y}=-\frac{\partial^{2} u_{3}}{\partial y^{2}}, \\
\kappa_{\theta}=\frac{1}{R^{2}}\left(\frac{\partial u_{2}}{\partial \theta}-\frac{\partial^{2} u_{3}}{\partial \theta^{2}}\right), & \kappa_{y \theta}=\frac{1}{R}\left(\frac{\partial u_{2}}{\partial y}-\frac{\partial^{2} u_{3}}{\partial y \partial \theta}\right) .
\end{array}
$$

Из вариационного уравнения (5) получаем необходимые уравнения динамики геометрически нерегулярной оболочки и краевые условия. Три уравнения динамики оболочки получаются, если в первом интеграле обратить в нуль коэффициенты при независимых вариациях. Остальные члены уравнения (5) определяют краевые условия задачи.

В случае осесимметричной деформации ребристой оболочки уравнения имеют вид [6]

$$
\frac{\partial N_{1}^{p}}{\partial y}-h_{0} \rho_{0} \frac{\partial^{2} u_{1}}{\partial t^{2}}\left(1+\sum_{j=1}^{n} k_{1 j} \Delta \Gamma_{y j}\right)=-q_{y}, \quad \frac{\partial^{2} M_{1}^{p}}{\partial y^{2}}-\frac{1}{R} N_{2}^{p}-h_{0} \rho_{0} \frac{\partial^{2} u_{2}}{\partial t^{2}}\left(1+\sum_{j=1}^{n} k_{1 j} \Delta \Gamma_{y j}\right)=-q_{n},
$$

где зависимости обобщенных сил $N_{1}^{p}, N_{2}^{p}$ и обобщенного момента $M_{1}^{p}$ от перемещений имеют вид

$$
\begin{gathered}
N_{1}^{p}=\frac{E h_{0}}{1-\mu_{0}^{2}}\left\{\left[\frac{\partial u_{1}}{\partial y}+\mu_{0} \frac{1}{R} u_{2}\right]\left(1+\sum_{j=1}^{n} k_{1 j} \Delta \Gamma_{y j}\right)-\frac{\partial^{2} u_{2}}{\partial y^{2}} h_{0} \sum_{j=1}^{n} k_{2 j} \Delta \Gamma_{y j}\right\}, \\
N_{2}^{p}=\frac{E h_{0}}{1-\mu_{0}^{2}}\left\{\left[\frac{1}{R} u_{3}+\mu_{0} \frac{\partial u_{1}}{\partial y}\right]\left(1+\sum_{j=1}^{n} k_{1 j} \Delta \Gamma_{y j}\right)-\mu_{0} \frac{\partial^{2} W}{\partial y^{2}} h_{0} \sum_{j=1}^{n} k_{2 j} \Delta \Gamma_{y j}\right\}, \\
M_{1}^{p}=-\frac{E h_{0}^{3}}{12\left(1-\mu_{0}^{2}\right)} \frac{\partial^{2} u_{3}}{\partial y^{2}}\left(1+\sum_{j=1}^{n} k_{3 j} \Delta \Gamma_{y j}\right)+\frac{E h_{0}}{1-\mu_{0}^{2}}\left(\frac{\partial u_{1}}{\partial y}+\mu_{0} \frac{1}{R} u_{3}\right) h_{0} \sum_{j=1}^{n} k_{2 j} \Delta \Gamma_{y j}
\end{gathered}
$$

и граничные условия для перемещений на торцах $\frac{\partial u_{1}}{\partial y}=0, u_{2}=0, \frac{\partial^{2} u_{3}}{\partial y^{2}}=0$ при $y=-l / 2$ и $y=l / 2$.

Тогда уравнения динамики ребристой оболочки в цилиндрической системе координат при осесимметричном деформации имеют вид

$$
\begin{gathered}
\frac{E h_{0}}{1-\mu_{0}^{2}} \frac{\partial}{\partial y}\left(\left[\frac{\partial u_{1}}{\partial y}-\mu_{0} \frac{u_{3}}{R}\right]\left(1+\sum_{j=1}^{n} k_{1 j} \Delta \Gamma_{y j}\right)+\frac{\partial^{2} u_{3}}{\partial y^{2}} h_{0} \sum_{j=1}^{n} k_{2 j} \Delta \Gamma_{y j}\right)= \\
=h_{0} \rho_{0} \frac{\partial^{2} u_{1}}{\partial t^{2}}\left(1+\sum_{j=1}^{n} k_{1 j} \Delta \Gamma_{y j}\right)-q_{y}, \\
\frac{\partial^{2}}{\partial y^{2}}\left(\frac{E h_{0}^{3}}{12\left(1-\mu_{0}^{2}\right)} \frac{\partial^{2} u_{3}}{\partial y^{2}}\left[1+\sum_{j=1}^{n} k_{3 j} \Delta \Gamma_{y j}\right]+\frac{E h_{0}}{1-\mu_{0}^{2}}\left[\frac{\partial u_{1}}{\partial y}-\mu_{0} \frac{u_{3}}{R}\right] h_{0} \sum_{j=1}^{n} k_{2 j} \Delta \Gamma_{y j}\right)+
\end{gathered}
$$




$$
\begin{gathered}
+\frac{1}{R} \frac{E h_{0}}{1-\mu_{0}^{2}}\left\{\left[\frac{u_{3}}{R}-\mu_{0} \frac{\partial u_{1}}{\partial y}\right]\left(1+\sum_{j=1}^{n} k_{1 j} \Delta \Gamma_{y j}\right)-\mu_{0} \frac{\partial^{2} u_{3}}{\partial y^{2}} h_{0} \sum_{j=1}^{n} k_{2 j} \Delta \Gamma_{y j}\right\}= \\
=-h_{0} \rho_{0} \frac{\partial^{2} u_{3}}{\partial t^{2}}\left(1+\sum_{j=1}^{n} k_{1 j} \Delta \Gamma_{y j}\right)+q_{n}
\end{gathered}
$$

где $k_{1 j}=\left(1-\frac{h_{0}}{h_{p j}}\right) \frac{h_{p j}}{h_{0}}, \quad k_{2 j}=\left(1-\frac{h_{0}}{h_{p j}}\right) \frac{h_{p j}^{2}}{2 h_{0}^{2}}, \quad k_{3 j}=\left(1-\frac{h_{0}}{h_{p j}}\right)\left(4-2 \frac{h_{0}}{h_{p j}}+\frac{h_{0}^{2}}{h_{p j}^{2}}\right) \frac{h_{p j}^{3}}{h_{0}^{3}}$.

Здесь $\Delta \Gamma_{y j}=\Gamma\left(y-y_{j}\right)-\Gamma\left(y-y_{j}-\varepsilon_{0 j}^{*}\right), \Gamma(y)-$ единичная функции Хевисайда по продольной координате $y ; y_{j}$ - точка появления ребра по продольной координате; $\mu_{0}-$ коэффициент Пуассона материала оболочки; $E$ - модуль Юнга материала оболочки; $\rho_{0}-$ плотность материала оболочки.

Таким образом, были получены уравнения динамики геометрически нерегулярной оболочки с ребрами жесткости ступенчато изменяющейся высоты.

Поверхностная нагрузка определяется напряжением со стороны жидкости

$$
q_{s}=-\left.\left[p_{r y} \cos \left(\bar{n}, \bar{n}_{r}\right)+p_{y y} \cos (\bar{n}, \bar{j})\right]\right|_{r=R_{2}+\delta+u_{3}}, \quad q_{n}=-\left.\left[p_{r r} \cos \left(\bar{n}, \bar{n}_{r}\right)+p_{r y} \cos (\bar{n}, \bar{j})\right]\right|_{r=R_{2}+\delta+u_{3}}
$$

где $\bar{n}-$ единичный вектор нормали к срединной поверхности оболочки; $\bar{s}-$ единичный вектор в продольном направлении в срединной поверхности оболочки, противоположный единичному вектору $\bar{j}$; $\bar{n}_{r}, \bar{j}-$ единичные векторы введенной цилиндрической системы координат,

$$
\begin{gathered}
k_{1 j}=\left(1-\frac{h_{0}}{h_{p j}}\right) \frac{h_{p j}}{h_{0}}, \quad k_{2 j}=\left(1-\frac{h_{0}}{h_{p j}}\right) \frac{h_{p j}^{2}}{2 h_{0}^{2}}, \quad k_{3 j}=\left(1-\frac{h_{0}}{h_{p j}}\right)\left(4-2 \frac{h_{0}}{h_{p j}}+\frac{h_{0}^{2}}{h_{p j}^{2}}\right) \frac{h_{p j}^{3}}{h_{0}^{3}}, \\
p_{r r}=-p+2 \nu \rho \frac{\partial V_{r}}{\partial r}, \quad p_{r y}=\nu \rho\left(\frac{\partial V_{r}}{\partial y}+\frac{\partial V_{y}}{\partial r}\right), \quad p_{y y}=-p+2 \nu \rho \frac{\partial V_{y}}{\partial y} \\
\cos \left(\bar{n}, \bar{n}_{r}\right)=\frac{R_{2}+\delta+u_{3}}{|\bar{N}|}, \quad \cos (\hat{n}, \bar{j})=-\frac{R_{2}+\delta+u_{3}}{|\bar{N}|} \frac{\partial u_{3}}{\partial y}, \quad|\bar{N}|=\left(R_{2}+\delta+u_{3}\right) \sqrt{1+\left(\frac{\partial u_{3}}{\partial y}\right)^{2}}
\end{gathered}
$$

Граничные условия уравнений представляют собой условия свободного (шарнирного) опирания:

$$
u_{3}=0, \quad \frac{\partial^{2} u_{3}}{\partial y^{2}}=0, \quad \frac{\partial u_{1}}{\partial y}=0 \quad \text { при } \quad y= \pm l / 2 .
$$

Таким образом, получили связанную задачу гидроупругости для круглой трубы кольцевого сечения с абсолютно жестким внутренним цилиндром и упругой, геометрически нерегулярной внешней оболочкой, свободно опираемой на концах трубы.

Выполнено при поддержке гранта Президента РФ (проект МД-1025.2012.8) и грантов РФФИ (проекты 12-01-31154-мол_а, 12-01-31161-мол_а).

\section{Библиографический список}

1. Башта Т. М. Машиностроительная гидравлика. М. : Машгиз, 1963. 696 с.

2. Могилевич Л. И., Андрейченко К. П. Динамика гироскопов с цилиндрическим поплавковым подвесом. Саратов : Изд-во Сарат. ун-та, 1987. 160 с.

3. Могилевич Л. И., Попов В. С. Прикладная гидроупругость в машино- и приборостроении / ФГОУ ВПО «Саратовский ГАУ». Саратов, 2003. 156 с.

4. Симдянкин A. A. Контактно-силовое взаимодействие деталей цилиндро-поршневой группы / ФГОУ ВПО «Саратовский ГАУ». Саратов, 2003. 144 с.

5. Кондратов Д. В., Могилевич Л. И. Математическое моделирование процессов взаимодействия двух цилиндрических оболочек со слоем жидкости между ними при свободном торцевом истечении в условия вибрации // Вестн. Сарат. гос. техн. ун-та. 2007. № 3(26), вып. 1. С. 22-31.

6. Могилевич Л. И., Попова А. А., Попов В. С. Динамика взаимодействия упругой цилиндрической оболочки с ламинарным потоком жидкости внутри нее применительно к трубопроводному транспорту // Наука и техника транспорта. 2007. № 2. С. 64-72. 


\title{
The Problem of a Hydroelasticity for a Tube Ring-type a Profile with Elastic, Geometrically Irregular Outer Shell at Pressure Influence
}

\author{
D. V. Kondratov ${ }^{1}$, Y. N. Kondratova ${ }^{2}$, V. S. Popov ${ }^{3}$, I. V. Plaksina ${ }^{4}$ \\ ${ }^{1}$ Stolypin Volga Region Institute Russian Academy of Public Administration under the President of the Russian Federation, Russia, \\ 410031, Saratov, Sobornaya st., 23/25, KondratovDV @ yandex.ru \\ ${ }^{2}$ Saratov State University, Russia, 410012, Saratov, Astrahanskaya st., 83, KondratovaUN@ info.sgu.ru \\ ${ }^{3}$ Saratov State Technical University, Russia, 410054, Saratov, Politekhnicheskaya st., 77, vic_p@bk.ru \\ ${ }^{4}$ Stolypin Volga Region Institute Russian Academy of Public Administration under the President of the Russian Federation, Russia, \\ 410031, Saratov, Sobornaya st., 23/25, chefirina@yandex.ru \\ The mechanical model presented in the form of a tube of ring section, formed by two surfaces of coaxial cylindrical shells cooperating \\ with viscous incompressible liquid is considered. The mathematical model of this system consisting of the differential equations \\ in private derivatives of describing dynamics of viscous incompressible liquid and an elastic ridge shell together with boundary \\ conditions is constructed.
}

Key words: hydroelasticity, viscous liquid, tube of ring section, geometrically irregular shell.

\section{References}

1. Bashta T. M. Mashinostroitel'naia gidravlika [Machine-building hydraulics]. Moscow, Mashgiz, 1963, 696 p. (in Russian).

2. Mogilevich L. I., Andrejchenko K. P. Dinamika giroskopov $s$ tsilindricheskim poplavkovym podvesom [Dynamics of gyroscopes with cylindrical floating suspension]. Saratov, Saratov Univ. Press, 1987, 160 p. (in Russian).

3. Mogilevich L. I., Popov V. S. Prikladnaia gidrouprugost' $v$ mashino- $i$ priborostroenii [Applied hydroelasticity in mechanical engineering and instrument making]. Saratov, 2003, 156 p. (in Russian).

4. Simdyankin A. A. Kontaktno-silovoe vzaimodeistvie detalei tsilindro-porshnevoi gruppy [The kontakt-force

interaction of details cylidr-piston group]. Saratov, 2003, 144 p. (in Russian).

5. Kondratov D. V., Mogilevich L. I. Mathematical modelling of processes of interaction of two cylindrical environments with the layer of the liquid between them under free leakage conditions of foundation vibration. Vestnik Saratovskogo gosudarstvennogo tekhnicheskogo universiteta, 2007, no. 3(26), iss. 1, pp. 22-31 (in Russian).

6. Mogilevich L. I., Popova A. A., Popov V. S. Dynamics's priests of interaction of an elastic cylindrical environment with a laminar stream of a liquid inside of it with reference to pipeline transport. Nauka i tekhnika transporta, 2007, no. 2, pp. 64-72 (in Russian).

УДК 501.1

\section{БИОМЕХАНИКА СОННОЙ АРТЕРИИ ЧЕЛОВЕКА С ПАТОЛОГИЧЕСКОЙ ИЗВИТОСТЬЮ}

\section{Л. Ю. Коссович ${ }^{1}$, К. М. Морозов ${ }^{2}$, О. Е. Павлова ${ }^{3}$}

\begin{abstract}
${ }^{1}$ Доктор фризико-математических наук, профрессор кафредры математической теории упругости и биомеханики, Саратовский государственный университет им. Н. Г. Чернышевского, nano-bio@sgu.ru

${ }^{2}$ Ведущий научный сотрудник отдела хирургии сосудов, Первый Московский государственный медицинский университет им. И. М. Сеченова, nano-bio@sgu.ru

${ }^{3}$ Инженер отдела биомеханики ОНИ наноструктур и биосистем, Саратовский государственный университет им. Н. Г. Чернышевского, Pavlovaoe@info.sgu.ru
\end{abstract}

Патологическая извитость сонной артерии занимает второе место в структуре причин сосудисто-мозговой недостаточности. Ранее авторами уже было описано влияние типа патологической извитости на поведение сонной артерии. В данной работе рассмотрено влияние различных анатомических (угол изгиба, размер ампулы) и реологических (уровень гематокрита) фракторов на гемодинамику и напряженно-десрормированное состояние сонной артерии с патологической извитостью. Выявлено, что уменьшение угла изгиба приводит к снижению объема крови, поступающей в мозг, и возможному фрормированию септального стеноза в области изгиба, а снижение уровня гематокрита способствует инициации процесса атерогенеза в зоне изгиба внутренней сонной артерии. Проведено численное моделирование реконструктивной операции на патологически извитой сонной артерии конкретного человека с атеросклеротическим поражением. Проведен расчет модели с предполагаемой геометрией сонной артерии данного пациента до возникновения патологий. Показано, что объемный кровоток после операции восстанавливается на 11\%, но не достигает значений для сонной артерии данного пациента в норме.

Ключевые слова: конечно-элементный анализ, патологическая извитость, сонная артерия, биомеханика. 